\title{
Citizen petition: Sexual side effects of SSRIs and SNRIs
}

\author{
Division of Dockets Management, \\ Department of Health and Human Services, \\ Food and Drug Administration, \\ 5630 Fishers Lane, Rm. 1061, \\ Rockville, MD 20852, USA
}

April 23, 2018

The undersigned submits this petition to request the Commissioner of Food and Drugs to immediately require the addition of boxed warnings, warnings, precautions, and highlights of prescribing information to the product label for all selective serotonin reuptake inhibitor (SSRI) and serotonin-norepinephrine reuptake inhibitor (SNRI) products, including branded and generic formulations.

These include, but are not limited to, citalopram (Celexa), desvenlafaxine (Pristiq), duloxetine (Cymbalta), escitalopram (Lexapro), fluoxetine (Prozac), paroxetine (Paxil), sertraline (Zoloft), venlafaxine (Effexor), and vortioxetine (Trintellix).

\section{A. Action requested}

Require the immediate revision of all SSRI and SNRI product labels (including branded and generic formulations) to warn of serious and severe risks, as follows:

1. Add WARNINGS, PRECAUTIONS, and HIGHLIGHTS OF PRESCRIBING INFORMATION to inform that the use of and withdrawal from SSRIs and SNRIs can result in genital anesthesia, pleasureless or weak orgasm, delayed or absent orgasm, loss of libido, erectile dysfunction, decreased vaginal lubrication, reduced nipple sensitivity, flaccid glans penis during erection, reduced response to sexual stimuli, and decreased capacity to experience sexual pleasure.

2. Add WARNINGS, PRECAUTIONS, and HIGHLIGHTS OF PRESCRIBING INFORMATION to inform that the use of and withdrawal from SSRIs and SNRIs can result in persistent genital arousal disorder (PGAD).

3. Add WARNINGS, PRECAUTIONS, HIGHLIGHTS OF PRESCRIBING INFORMATION, and a BOXED WARNING to inform that sexual side effects can sometimes persist for years or indefinitely after discontinuation of the drug; they can emerge on treatment and remain afterwards, or emerge or worsen when the drug is stopped.

4. Send all manufacturers of SSRIs and SNRIs a notification letter of the need for a Risk Evaluation and Mitigation Strategy Plan to include the following:

i. Require manufacturers to send a Dear Health Care Provider Letter to psychiatrists, psychologists, internists, family practitioners, urologists and endocrinologists that informs the health care providers that the drug has serious adverse reactions associated with its use, and poses a significant public health concern. 
ii. Require manufacturers to develop a Medication Guide and Communication Plan to make patients aware that the drug has serious risks that could affect the patients' decision to use or continue to use the product.

\section{B. Statement of grounds}

SSRI and SNRI antidepressants are well known to cause adverse sexual effects. This was first established in healthy volunteer phase 1 trials in the 1980s. Current product labeling warns of disturbances to sexual functioning, but these warnings are insufficient.

\section{B.1. Genital anesthesia}

The ability of a serotonin reuptake inhibitor to reduce genital sensation was identified as far back as 1991. A study investigated the use of clomipramine as a possible treatment for premature ejaculation [1]. Neurophysiological testing was used to assess penile sensory threshold before and after treatment. While clomipramine is classed as a tricyclic antidepressant, it is a potent serotonin reuptake inhibitor.

Electrodes were placed on the subject's penis and very small electric currents were generated until the subject could feel the sensation. The current was then decreased until it could no longer be perceived. This second figure was taken as a measurement of penile sensory threshold. The study found that the drug had increased penile sensory threshold from $24.4 \mathrm{~V}$ before treatment to $30.2 \mathrm{~V}(24 \%)$ at the end of 30 days.

In 1999, a similar study involving fluoxetine found an increase in penile sensory threshold from $4.9 \mathrm{~mA}$ before treatment to $6.1 \mathrm{~mA}(24 \%)$ after one month [2]. The fluoxetine study also had the benefit of a control group (placebo) for whom penile sensory threshold remained unchanged.

In addition, there were multiple case reports of genital anesthesia during the 1990s linked to each of the major SSRIs [3-9], including one that involved reduced nipple sensitivity [8]. These date from 1991 to 2002, and involve the use of fluoxetine, sertraline, and paroxetine.

Clinical experience suggests that almost everyone who takes an SSRI or SNRI experiences a degree of reduced genital sensitivity, often occurring within 30 minutes of taking the first dose. In this sense, almost everyone taking a serotonin reuptake inhibitor has altered sexual functioning.

Despite this, and the fact there is an indication for a serotonin reuptake inhibitor for premature ejaculation based on this effect (ie. dapoxetine), genital anesthesia remains completely absent from SSRI and SNRI product labels.

\section{B.2. Persistent sexual side effects after stopping medication}

The common assumption that sexual functioning typically returns to pre-drug baseline after the use of an SSRI or SNRI has no clear basis. While clinical experience points to some recovery of function in many people, there is no robust evidence that anyone who takes an SSRI or SNRI actually recovers $100 \%$ of their original genital sensation, sexual response and capacity to experience sexual pleasure. This has never been properly investigated.

Pre-medication sexual functioning baselines are rarely taken; there are no queries in any standard sexual functioning instruments related to genital numbness or orgasmic anhedonia; there is rarely any follow-up, and no-one has systematically asked whether the sexual side effects have resolved - or if anyone has, the data has not been published. 
In 2006, a case report from Bolton et al appeared in the medical literature describing a 26-year-old male with persistent sexual side effects following treatment with sertraline [10]. Sexual dysfunction had emerged early in treatment, which was continued for around four or five months. Symptoms of genital anesthesia, pleasureless orgasm, delayed orgasm, and loss of libido had persisted for six years after withdrawal of the drug.

The subject was in no doubt that sertraline was responsible, and the authors of the case report acknowledged having been torn, but they tentatively opted for a psychodynamic interpretation of the ongoing symptoms because they found nothing in the literature at that time to support the notion of enduring SSRI sexual side effects. However, they noted that an enduring pharmacological effect could not be ruled out as an alternative explanation.

Three case reports followed from Csoka and Shipko [11].

- A 24-year-old male experienced a persistent loss of libido, and severe tactile insensitivity of his penis, chest and abdomen after withdrawal of citalopram.

- A 27-year-old female who described having a very high libido since puberty, developed a dramatic loss of libido, reduced genital and nipple sensitivity, and anorgasmia within three days of using fluoxetine. After discontinuing the drug seven months later, orgasmic capacity returned but at a dramatically reduced intensity and with a refractory period of several days. Loss of libido remained and tactile sensitivity only partially returned.

- A 30-year-old male experienced a severe drop in sexual desire, moderate erectile dysfunction, difficulty reaching orgasm with a long refractory time, reduced ejaculate volume, and genital anesthesia within four to five days of starting sertraline. The drug was discontinued after five weeks, but the problems remained unchanged several years later. Sildenafil, supplemental testosterone, and herbal remedies were tried but without success.

Bahrick described post-SSRI sexual dysfunction (PSSD) as an iatrogenic condition in which sexual side effects fail to resolve to baseline after discontinuation of an SSRI/SNRI, often characterized by features of genital anesthesia and pleasureless orgasm [12]. She noted that these are not typical features of sexual dysfunction, and are not associated with conditions for which antidepressants are prescribed.

The article also drew attention to a 1999 study by Montejo et al which inadvertently appeared to discover evidence of post-treatment changes to sexual function [13]. A group of patients who were experiencing sexual side effects on an SSRI were switched to the dopaminergic antidepressant, amineptine. Six months after the switch, 55\% still had at least some type of sexual dysfunction compared to only $4 \%$ in the control group who were treated with amineptine alone and were not exposed to an SSRI.

In 2007, Kauffman and Murdock reported the case of a 32-year-old female who developed genital anesthesia, diminished orgasmic intensity, difficulty achieving orgasm, and a substantial decrease in libido within days of starting citalopram [14]. She described her genital region as "feeling totally numb". All of these difficulties remained essentially unchanged after discontinuation of the drug, with the subject continuing to experience low libido, minimal genital tactile sensation, and orgasmic dysfunction. Physical and psychological testing did not reveal a cause.

In 2008, three more cases of persistent sexual dysfunction following SSRIs were reported by Csoka et al. [15].

- A 29-year-old male developed severe erectile dysfunction and loss of nocturnal and morning erections within 3-4 days of using fluoxetine, which persisted for 11 years since discontinuing the drug. He was 
left only able to achieve penetrative sex by using prescribed alprostadil. No psychological or physical health problems were found after consultations with two family practice physicians, three urologists, two neurologists, an endocrinologist, and a radiologist.

- A 44-year-old male described persistent loss of libido, genital anesthesia, ejaculatory anhedonia, and erectile dysfunction since discontinuing citalopram. No physical or psychological cause was found after consultations with several health care professionals, including an endocrinologist, urologist, and psychologist.

- A 28-year-old male developed loss of libido, genital anesthesia, and ejaculatory anhedonia on several different SSRIs, which persisted after discontinuing the medication. Numerous tests failed to reveal any physical or biochemical abnormalities. Psychological factors were ruled out by three different psychiatrists.

A review article published by Bahrick in the same year highlighted the significance of two large placebo controlled studies into the use of SSRIs as a treatment for premature ejaculation [16]. The studies by Safarinejad and Hosseini [17], and by Arafa and Shamloul [18], found that the ejaculation-delaying effects of citalopram and sertraline, respectively, persisted for a significant number of participants at 3and 6-month follow-up after the drugs had been withdrawn. A similar study by Safarinejad involving escitalopram has since been published with the same outcome [19].

A study by Tanrikut et al which aimed to investigate the effects of paroxetine on sperm also assessed sexual function before, during, and after the five-week treatment period [20]. It was revealed that brief sexual function inventory (BSFI) scores for erectile and ejaculatory functions had not returned to baseline four weeks after discontinuation of the drug, with $9 \%$ of patients complaining of more than mild dysfunction.

Of note, SSRIs have been shown to have adverse effects on semen quality and sperm DNA fragmentation, which may be detrimental to male fertility [21-24].

There were published calls for epidemiological studies to investigate the prevalence of PSSD, from Kauffman in 2008 [25], and from Farnsworth and Dinsmore in 2009 [26].

In 2011, the US Prozac product information [27] was amended to warn that:

"Symptoms of sexual dysfunction occasionally persist after discontinuation of fluoxetine treatment."

In 2012, the Netherlands Pharmacovigilance Center (Lareb) published a report with details of 19 suspected cases of PSSD from their database [28]. The findings were subsequently published in the medical literature by Ekhart and van Puijenbroek [29]. The drugs involved were paroxetine, sertraline, venlafaxine, citalopram, fluoxetine, fluvoxamine, and escitalopram. Fifteen reports came from consumers, with the remainder from general practitioners, a pharmacist, and a pharmaceutical company. There were 13 male subjects and six female, aged from 20 to 59 years. Duration of treatment ranged from 9 days to 10 years, and the time since stopping the drug and still experiencing sexual side effects ranged from two months to two years.

In 2013, the fifth edition of the Diagnostic and Statistical Manual of Mental Disorders (DSM-5) was published. The section on Substance/Medication-Induced Sexual Dysfunction [30] states:

"In some cases, serotonin reuptake inhibitor-induced sexual dysfunction may persist after the agent is discontinued."

Also in 2013, Stinson authored a qualitative study on the impact of persistent sexual side effects of selective serotonin reuptake inhibitors after discontinuing treatment [31]. Four male and five female PSSD sufferers 
were assessed, aged from 22 to 59 years. The drugs involved were citalopram, escitalopram, fluoxetine, paroxetine, and sertraline. Symptoms included genital anesthesia, orgasmic anhedonia, anorgasmia, erectile dysfunction, and hypoactive desire. Six of the subjects reported having the condition for over a year since stopping the medication, with the longest being three years and nine months.

In 2014, Hogan et al published a study of 120 cases of enduring sexual dysfunction, including 90 involving serotonin reuptake inhibitors. The article drew attention to commonalities between the enduring problems following SSRIs, SNRIs, finasteride, and isotretinoin [32].

Also in 2014, Waldinger et al. offered the first evidence that the genital anesthesia in PSSD may involve a peripheral component [33]. A 43-year-old male developed penile anesthesia and scrotum hypesthesia on paroxetine which persisted after stopping the drug. He had attempted to assess the level of insensitivity by applying Tiger Balm to his penis, but felt nothing except a vague sensation over his scrotum. A small degree of tactile sensitivity was regained following treatment with low-power laser irradiation, though there was no improvement in sexual responsiveness. It was hypothesized that SSRIs may disrupt the normal functioning of transient receptor potential (TRP) ion channels.

In 2015, Ben-Sheetrit et al published a study of 183 possible cases of PSSD including 23 high-probability cases from an online survey [34]. The high-probability cases involved fluoxetine $(n=6)$, citalopram $(n=5)$, escitalopram $(n=5)$, venlafaxine $(n=3)$, sertraline $(n=2)$, desvenlafaxine $(n=1)$, and paroxetine $(n=1)$. There were 19 male subjects and four female, aged from 21 to 47 years, all of whom had normal scores on depression and anxiety scales, and did not have confounding conditions, medications, or addictive substance use. The shortest duration of treatment was 4 days of sertraline. The longest time since stopping an SSRI and still experiencing sexual side effects was 16 years.

In 2017, two review articles about PSSD were published by Reisman [35] and Bala et al [36]. Both advised that patients should be warned about PSSD when using SSRIs.

In the same year, Muquebil Ali Al Shaban Rodríguez reported the case of a young male with decreased libido and erectile dysfunction which had persisted for 12 weeks after withdrawal of paroxetine [37]. The subject had no concurrent physical illness, no medication or recreational drug use, and was free of affective symptoms. The authors concluded that the previous use of paroxetine was the most likely cause.

A letter (available on request) from Dr. A. Nathwani (Chief Medical Officer / Global Head of Medical Function / Executive Vice President of Sanofi), dated December 4, 2017, reported that following an internal investigation encompassing a review of the Sanofi global safety database, available literature, pharmacovigilance text books and Sanofi's Generic Core Data Sheets (GCDS), it was determined that:

"The cumulative weighted evidence gathered for the mentioned SSRIs was sufficient to conclude that these products could be associated with persistent sexual disorders after treatment discontinuation."

The "mentioned SSRIs" were fluoxetine, paroxetine, citalopram, escitalopram, and sertraline.

In a letter (available on request) dated January 15, 2018, the Medicines and Healthcare products Regulatory Agency (MHRA) confirmed that up to January 5, 2018, they had received a total of 1475 UK spontaneous suspected ADR reports of sexual dysfunction associated with SSRIs. Of these, 309 indicated that the sexual dysfunction persisted after discontinuation of the drug, and in 963 cases it was unknown whether the reaction continued after the drug was withdrawn.

In February 2018, a review article about PSSD by Coskuner et al raised concerns about the possibility of long-term sexual consequences for people exposed to SSRIs during pregnancy or at a young age [38]. 
In May 2018, Healy et al published a study of 300 cases of enduring sexual dysfunction of which 221 were after the previous use of serotonin reuptake inhibitors [39]. These were sourced from RxISK's adverse event reporting system.

- Reports involving serotonin reuptake inhibitors were received from 34 countries. There were 171 male subjects and 50 female, aged from 15 to 66 years.

- The reported drugs were escitalopram $(n=42)$, citalopram $(n=41)$, paroxetine $(n=40)$, sertraline $(n=32)$, fluoxetine $(n=31)$, venlafaxine $(n=19)$, duloxetine $(n=10)$, fluvoxamine $(n=2)$, vortioxetine $(n=2)$, clomipramine $(n=1)$, and desvenlafaxine $(n=1)$. Fifteen subjects reported that the duration of drug exposure had been less than two weeks.

- Symptoms included loss of libido $(n=171)$, erectile dysfunction $(n=147)$, genital anesthesia $(n=114)$, pleasureless or weak orgasm $(n=86)$, loss of nocturnal erections $(n=22)$, vaginal dryness/pain $(n=9)$, and reduced nipple sensitivity $(n=6)$. Four subjects reported an erectile abnormality in which the glans penis remained flaccid when the shaft was erect.

- Forty-one reports involved adverse sexual effects that either emerged or became significantly worse upon stopping a serotonin reuptake inhibitor. This has similarities to antipsychotic-induced tardive dyskinesia which can appear on treatment and remain afterwards, or only appear when the medication is stopped.

- Eight subjects reported that the time since stopping a serotonin reuptake inhibitor and still experiencing sexual side effects was over 10 years, including one over 20 years.

\section{B.3. Animal studies}

Treatment with fluoxetine has been shown to cause persistent desensitization of 5-HT1A receptors after removal of the SSRI in rats [40].

Rodent studies have shown that treatment with SSRIs at a young age resulted in permanently decreased sexual behavior in adulthood [41-43], including the presence of long-term neurological changes [41]. Maternal exposure to fluoxetine was also found to impair sexual motivation in adult male mice [44].

In 2016, Simonsen et al published a systematic review of 14 animal studies measuring sexual behavior after discontinuation of treatment with SSRIs [45]. It concluded:

"Our results showed substantial and lasting effects on sexual behaviour in rats after exposure to an SSRI early in life on important sexual outcomes."

\section{B.4. Persistent genital arousal disorder (PGAD)}

PGAD is a condition involving an uncontrollable and relentless feeling of physiological arousal in the genitals, but without any accompanying feeling of sexual desire. It is the mirror image of PSSD. The use of SSRIs or SNRIs, and often their withdrawal, has consistently been reported as one of the triggers of the condition, which can endure for years after the medication has been discontinued. Desperate women with PGAD have resorted to treatment with clitoridectory, pudendal nerve ablation, electroconvulsive therapy (ECT), and other drastic measures.

The condition was first described by Leiblum and Nathan in 2001, and was originally called persistent sexual arousal syndrome (PSAS) [46].

A letter to the editor of the Journal of Sexual Medicine published in 2005, described a link between SSRIs and PGAD [47]. The author attributed the onset of her own condition to the taking and withdrawal of SSRIs, and reported that a significant number of people in an online PGAD support group to which 
she belonged had also used and discontinued SSRIs, namely paroxetine $(n=4)$, citalopram $(n=5)$, and fluoxetine $(n=1)$.

In 2006, Goldmeier, Bell, and Richardson hypothesized that the onset of PGAD following SSRI withdrawal may involve an increase in atrial natriuretic peptide [48].

Later that year, Goldmeier and Leiblum noted that within a small study group consisting of physicians and PGAD sufferers, several people had reported that their unprovoked genital arousal began when an SSRI was discontinued [49].

In 2007, Mahoney and Zarate described a 32-year-old female who developed unrelenting and unwanted sensations of genital arousal when her dose of venlafaxine was increased [50]. These sensations remitted upon lowering the dose. The authors noted that her symptoms matched the five criteria of PGAD listed by Leiblum and Nathan in 2001.

In 2008, five case reports by Leiblum and Goldmeier were published linking SSRIs to the onset of PGAD. These involved the use of venlafaxine $(n=3)$, fluoxetine, $(n=1)$, and sertraline $(n=1)$. All five cases involved female subjects. In four of the cases, development of the condition was specifically linked to withdrawal of the drug [51].

In a 2009 study by Waldinger et al, 13\% $(n=3)$ of patients with PGAD who visited a neurosexology outpatient department had been taking SSRIs before onset of the condition, two of whom developed it during or shortly after discontinuation of the drug [52].

In 2014, Eibye and Jensen reported the case of a 31-year-old woman who developed PGAD upon withdrawal of paroxetine [53]. A pelvic MRI revealed a tarlov cyst, but its size and location could not explain the patient's symptoms. A variety of treatments were tried including ECT, but with only temporary improvement.

In 2015, de Magalhães and Kumar described a 57-year-old female who developed PGAD following discontinuation of citalopram [54].

A 2017 study by Jackowich et al using a detailed online survey found that $20 \%(n=23)$ of subjects attributed the onset of their condition to the previous use of SSRIs [55].

The aforementioned 2018 paper from Healy et al included six reports of PGAD linked to the previous use of serotonin reuptake inhibitors [39].

Note that FDA do not currently have codes for PGAD or PSSD.

\section{B.5. Other drugs}

FDA updated the product information for finasteride products in 2011 to warn of persisting sexual side effects after discontinuation of treatment, with further warnings added in 2012 [56]. A similar posttreatment sexual dysfunction following isotretinoin has been reported in the literature [32, 39].

\section{B.6. Companies}

In September 2017, Healy wrote to all companies producing serotonin reuptake inhibitors, drawing their attention to the existence of these problems on medicines that have originated with them. Some explored the issue of seeking further details on the patients, but have not followed up on this. 


\section{B.7. Conclusion}

The data make it clear that SSRIs and SNRIs are potent disruptors of sexual function, and that adverse sexual effects can sometimes persist for years or indefinitely after discontinuation of the drug. In some cases, these effects only emerge or worsen when the drug is withdrawn. It also appears that post-treatment problems can occur after only a brief exposure to the drug.

At present, the US Prozac product sheet is the only one to mention that sexual side effects can persist after the drug is stopped, though the wording is inadequate. The labeling for duloxetine makes no mention of a comparable problem, despite being produced by the same company.

PSSD and PGAD can be life-changing, making it difficult or impossible to engage in normal intimate relationships, or even function in daily life.

Current labeling does not adequately convey the breadth, severity or potentially permanent nature of the adverse sexual effects from SSRI and SNRI products. In particular, genital anesthesia and pleasureless orgasm should be mentioned specifically because these are highly unusual effects and not typical features of sexual dysfunction. Neither patients or health care professionals can reasonably be expected to know that the impact on sexual functioning could include profound genital numbness and the loss of ability to experience pleasure during orgasm.

Across the literature, a number of different terms are used to describe the altered quality of orgasm in PSSD, such as pleasureless orgasm, ejaculatory anhedonia, orgasmic anhedonia, and diminished orgasmic intensity. However, they are all describing the same phenomenon ie. an orgasm in men and women which is markedly lacking in pleasurable sensation, and feels profoundly and abnormally muted to the sufferer, such that it is rendered little more than a series of rhythmic muscle contractions in the genital area.

Without adequate warnings about the risk of potentially permanent damage to sexual functioning, patients are being deprived of informed consent. It is currently impossible for patients and health care professionals to weigh the benefits of treatment against the harms. We therefore request that clear warnings are immediately added to all SSRI and SNRI products.

\section{Environmental impact}

We claim categorical exclusion from the environmental assessment requirement under 21 C.F.R §25.31(a), as the requested action would not increase the use of the active moiety.

\section{Economic impact}

We will submit an economic impact analysis upon request, if this is deemed to be necessary.

\section{E. Certification}

The undersigned certifies that, to the best knowledge and belief of the undersigned, this petition includes all information and views on which the petition relies, and that it includes representative data and information known to the petitioner which are unfavorable to the petition. 
Address for correspondence:

Professor David Healy

Data Based Medicine Americas Ltd.

95 Sandringham Drive

Toronto, Ontario

Canada

M3H 1E1

Email: david.healy@rxisk.org

Respectfully submitted,

Audrey S. Bahrick, PhD

Staff Psychologist

University Counseling Service, The University of Iowa, Iowa City, Iowa, USA

Joseph Ben-Sheetrit, MD

Psychiatry Resident

Clalit Health Services, Israel

Antonei B. Csoka, $\mathrm{PhD}$

Assistant Professor

Epigenetics Laboratory, Department of Anatomy, Howard University, Washington, DC, USA

Pia Brandt Danborg, MSc

PhD Fellow

Nordic Cochrane Centre, Copenhagen, Denmark

Faculty of Health and Medical Sciences, University of Copenhagen, Denmark

Keith D. Farnsworth, PhD, MSc (Public Health and Epidemiology), BSc

Lecturer in Theoretical Biology

Queen's University Belfast, UK

David Goldmeier, MD, FRCP

Consultant GU/Sexual Medicine

Jefferiss Wing, St Mary's Hospital, London, UK

Irwin Goldstein, MD

Director, San Diego Sexual Medicine, San Diego, CA, USA

Director, Sexual Medicine, Alvarado Hospital, San Diego, CA, USA

Clinical Professor of Surgery, University of California at San Diego, San Diego, CA, USA

Editor-in-Chief, Sexual Medicine Reviews

Peter C. Gøtzsche, MD, DSc

Professor and Director

Nordic Cochrane Centre, Copenhagen, Denmark 
David Healy, MD, FRCPsych

Professor of Psychiatry

North Wales Department of Psychological Medicine, Bangor, Wales, UK

Wayne J. G. Hellstrom, MD, FACS

Professor of Urology; Chief, Section of Andrology

Tulane University School of Medicine, New Orleans, LA, USA

Manoj Therayil Kumar, MD, FRCPsych

Director, Institute for Mind and Brain

InMind campus, Thrissur, Kerala, India

Joanna Le Noury, PhD

Senior Research Psychologist

North Wales Department of Psychological Medicine, Bangor, Wales, UK

Dee Mangin, MBChB, DPH, FRNZCGP

Professor, Associate Chair and Director of Research

Department of Family Medicine, McMaster University, Ontario, Canada

Omar Walid Muquebil Ali Al Shaban Rodríguez, MD

Psychiatrist and Family Doctor

Hospital Universitario San Agustín, Avilés, Asturias, Spain

Jalesh N. Panicker MD, DM, FRCP

Consultant Neurologist and Reader in Uro-neurology

The National Hospital for Neurology and Neurosurgery, Queen Square, London, UK

UCL Institute of Neurology, Queen Square, London, UK

Leah Pink, MN, NP-Adult

Nurse Practitioner, Wasser Pain Management Centre, Ontario, Canada

Adjunct Lecturer, Lawrence S. Bloomberg Faculty of Nursing, University of Toronto, Canada

Dave H. Schweitzer, MD, PhD

Department of Internal Medicine and Endocrinology

Reinier the Graaf Gasthuis, Delft, The Netherlands

Stuart Shipko, MD

Board Certified Psychiatrist

Private Practice, Pasadena, California, USA

Anders Lykkemark Simonsen, MD

Nordic Cochrane Centre

Copenhagen, Denmark

Rebecca D. Stinson, PhD

Licensed Psychologist

Minneapolis VA Health Care System, USA 
Barbora Vašečková, $\mathrm{MD}, \mathrm{PhD}$

Psychiatric Clinic, Slovak Medical University, Bratislava, Slovakia

Psychiatry Outpatient Clinics, University Hospital and Policlinic, The Brothers of Saint John of God in Bratislava, Bratislava, Slovakia

Abraham Weizman, MD

Professor of Psychiatry

Sackler Faculty of Medicine, Tel Aviv University, Israel

\section{References}

[1] Colpi GM, Fanciullacci F, Aydos K, Grugnetti C. Effectiveness mechanism of chlomipramine by neurophysiological tests in subjects with true premature ejaculation. Andrologia. 1991;23(1):45-7.

[2] Yilmaz U, Tatlişen A, Turan H, Arman F, Ekmekçioğlu O. The effects of fluoxetine on several neurophysiological variables in patients with premature ejaculation. J Urol. 1999;161(1):107-11.

[3] Neill JR. Penile anesthesia associated with fluoxetine use. Am J Psychiatry. 1991;148:1603.

[4] Measom MO. Penile anaesthesia and fluoxetine. Am J Psychiatry. 1992;149:709.

[5] King VL, Jr, Horowitz IR. Vaginal anesthesia associated with fluoxetine use. Am J Psychiatry. 1993;150:984-5.

[6] Ellison JM, DeLuca P. Fluoxetine-induced genital anesthesia relieved by Ginkgo biloba extract. J Clin Psychiatry. 1998;59:199-200.

[7] Diesenhammer EA, Trawoger R. Penile anesthesia associated with sertraline use. J Clin Psychiatry. 1999;60:869-70.

[8] Michael A, Mayer C. Fluoxetine-induced anaesthesia of vagina and nipples. Br J Psychiatry. 2000;176:299.

[9] Michael A, Andrews S. Paroxetine-induced vaginal anaesthesia. Pharmacopsychiatry. 2002;35:150-1.

[10] Bolton JM, Sareen J, Reiss JP. Genital anaesthesia persisting six years after sertraline discontinuation. J Sex Marital Ther. 2006;32(4):327-30.

[11] Csoka AB, Shipko S. Persistent sexual side effects after SSRI discontinuation. Psychother Psychosom. 2006;75(3):187-8.

[12] Bahrick AS. Post SSRI sexual dysfunction. American Society for the Advancement of Pharmacotherapy. Tablet 2006;7(3):2-3,10-11.

[13] Montejo AL, Llorca G, Izquierdo JA, Carrasco JL, Daniel E, Pérez-Sola V, Vicens E, Bousoño M, Sánchez-Iglesias S, Franco M, Cabezudo A, Rubio V, Ortega MA, Puigdellivol M, Domenech JR, Allué B, Sáez C, Mezquita B, Gálvez I, Pacheco L, de Miguel E. Sexual dysfunction with antidepressive agents. Effect of the change to amineptine in patients with sexual dysfunction secondary to SSRI. Actas Esp Psiquiatr. 1999;27(1):23-34.

[14] Kauffman RP, Murdock A. Prolonged Post-Treatment Genital Anesthesia and Sexual Dysfunction Following Discontinuation of Citalopram and the Atypical Antidepressant Nefazodone. The Open Women' Health Journal. 2007;1:1-3.

[15] Csoka AB, Bahrick A, Mehtonen OP. Persistent sexual dysfunction after discontinuation of selective serotonin reuptake inhibitors. J Sex Med. 2008;5(1):227-33.

[16] Bahrick AS. Persistence of sexual dysfunction side effects after discontinuation of antidepressant medications: Emerging evidence. The Open Psychology Journal. 2008;1:42-50.

[17] Safarinejad MR, Hosseini SY. Safety and efficacy of citalopram in the treatment of premature ejaculation: a double-blind placebo-controlled, fixed dose, randomized study. Int J Impot Res. 2006;18(2):164-9.

[18] Arafa M, Shamloul R. Efficacy of sertraline hydrochloride in treatment of premature ejaculation: a placebo-controlled study using a validated questionnaire. Int J Impot Res. 2006;18(6):534-8. 
[19] Safarinejad MR. Safety and efficacy of escitalopram in the treatment of premature ejaculation: a double-blind, placebo-controlled, fixed-dose, randomized study. J Clin Psychopharmacol. 2007;27(5):444-50.

[20] Tanrikut C, Feldman AS, Altemus M, Paduch DA, Schlegel PN. Adverse effect of paroxetine on sperm. Fertil Steril. 2010;94(3):1021-6.

[21] Tanrikut C, Schlegel PN. Antidepressant-associated changes in semen parameters. Urology. 2007;69(1):185.e5-7.

[22] Safarinejad MR. Sperm DNA damage and semen quality impairment after treatment with selective serotonin reuptake inhibitors detected using semen analysis and sperm chromatin structure assay. J Urol. 2008;180(5):2124-8.

[23] Koyuncu H, Serefoglu EC, Yencilek E, Atalay H, Akbas NB, Sarıca K. Escitalopram treatment for premature ejaculation has a negative effect on semen parameters. Int J Impot Res. 2011;23(6):257-61.

[24] Akasheh G, Sirati L, Noshad Kamran AR, Sepehrmanesh Z. Comparison of the effect of sertraline with behavioral therapy on semen parameters in men with primary premature ejaculation. Urology. 2014;83(4):800-4.

[25] Kauffman RP. Persistent Sexual Side Effects after Discontinuation of Psychotropic Medications. Primary Psychiatry. 2008.

[26] Farnsworth KD, Dinsmore WW. Persistent sexual dysfunction in genitourinary medicine clinic attendees induced by selective serotonin reuptake inhibitors. Int J STD AIDS. 2009;20(1):68-9.

[27] Lilly. US Prozac product information, p. 14 [Internet]. Available from: http://pi.lilly.com/us/prozac. pdf. Accessed 2017 May 14.

[28] Lareb, Netherlands Pharmacovigilance Center. SSRIs and persistent sexual dysfunction [Internet]. 2012. Available from: http://databankws.lareb.nl/Downloads/KWB_2012_3_SSRI.pdf. Accessed 2017 June 1.

[29] Ekhart GC, van Puijenbroek EP. Does sexual dysfunction persist upon discontinuation of selective serotonin reuptake inhibitors? Tijdschr Psychiatr. 2014;56(5):336-40.

[30] American Psychiatric Association: Diagnostic and Statistical Manual of Mental Disorders, 5th ed. Arlington, VA: American Psychiatric Association; 2013. p. 449.

[31] Stinson RD. The impact of persistent sexual side effects of selective serotonin reuptake inhibitors after discontinuing treatment: a qualitative study. PhD (Doctor of Philosophy) thesis, University of Iowa [Internet]. 2013. Available from: http://ir.uiowa.edu/etd/5061/. Accessed 2018 February 19.

[32] Hogan C, Le Noury J, Healy D, Mangin D. One hundred and twenty cases of enduring sexual dysfunction following treatment. Int J Risk Saf Med. 2014;26(2):109-16.

[33] Waldinger MD, van Coevorden RS, Schweitzer DH, Georgiadis J. Penile anesthesia in Post SSRI Sexual Dysfunction (PSSD) responds to low-power laser irradiation: a case study and hypothesis about the role of transient receptor potential (TRP) ion channels. Eur J Pharmacol. 2015;753:263-8.

[34] Ben-Sheetrit J, Aizenberg D, Csoka AB, Weizman A, Hermesh H. Post-SSRI Sexual Dysfunction: Clinical Characterization and Preliminary Assessment of Contributory Factors and Dose-Response Relationship. J Clin Psychopharmacol. 2015;35(3):273-8.

[35] Reisman Y. Sexual Consequences of Post-SSRI Syndrome. Sex Med Rev. 2017;5(4):429-33.

[36] Bala A, Tue Nguyen HM, Hellstrom WJG. Post-SSRI Sexual Dysfunction: A Literature Review. Sex Med Rev. 2018;6(1):29-34.

[37] Muquebil Ali Al Shaban Rodríguez OW, Álvarez de Morales Gómez-Moreno E, Fernández Fernández J, Fresno García C, del Mar Fernández Fernández M. Disfunción sexual persistente tras el tratamiento con inhibidores selectivos de la recaptación de serotonina: a propósito de un caso tras la retirada de paroxetina. Psiquiatría Biológica. 2017;24(2):70-2.

[38] Coskuner ER, Culha MG, Ozkan B, Kaleagasi EO. Post-SSRI Sexual Dysfunction: Preclinical to Clinical. Is It Fact or Fiction? Sex Med Rev. 2018;6(2):217-23. 
[39] Healy D, Le Noury J, Mangin D. Enduring Sexual Dysfunction after Treatment with Antidepressants, 5 $\alpha$-Reductase Inhibitors and Isotretinoin: 300 Cases. Int J Risk Saf Med. 2018. doi:10.3233/JRS180744.

[40] Raap DK, Garcia F, Muma NA, Wolf WA, Battaglia G, van de Kar LD. Sustained desensitization of hypothalamic 5-Hydroxytryptamine1A receptors after discontinuation of fluoxetine: inhibited neuroendocrine responses to 8-hydroxy-2-(Dipropylamino)Tetralin in the absence of changes in Gi/ o/z proteins. J Pharmacol Exp Ther. 1999;288(2):561-7.

[41] Maciag D, Simpson KL, Coppinger D, Lu Y, Wang Y, Lin RC, Paul IA. Neonatal Antidepressant Exposure has Lasting Effects on Behavior and Serotonin Circuitry. Neuropsychopharmacology. 2006;31(1):47-57.

[42] de Jong TR, Snaphaan LJ, Pattij T, Veening JG, Waldinger MD, Cools AR, Olivier B. Effects of chronic treatment with fluvoxamine and paroxetine during adolescence on serotonin-related behavior in adult male rats. Eur Neuropsychopharmacol 2006;16(1):39-48.

[43] Iñiguez SD, Warren BL, Bolaños-Guzmán CA. Short- and long-term functional consequences of fluoxetine exposure during adolescence in male rats. Biol Psychiatry. 2010;67(11):1057-66.

[44] Gouvêa TS, Morimoto HK, de Faria MJ, Moreira EG, Gerardin DC. Maternal exposure to the antidepressant fluoxetine impairs sexual motivation in adult male mice. Pharmacol Biochem Behav. 2008;90(3):416-9.

[45] Simonsen AL, Danborg PB, Gøtzsche PC. Persistent sexual dysfunction after early exposure to SSRIs: Systematic review of animal studies. Int J Risk Saf Med. 2016;28(1):1-12.

[46] Leiblum SR, Nathan SG. Persistent sexual arousal syndrome: a newly discovered pattern of female sexuality. J Sex Marital Ther. 2001;27(4):365-80.

[47] Freed L. Persistent sexual arousal syndrome. J Sex Med. 2005;2:743

[48] Goldmeier D, Bell C, Richardson D. Withdrawal of selective serotonin reuptake inhibitors (SSRIs) may cause increased atrial natriuretic peptide (ANP) and persistent sexual arousal in women? J Sex Med. 2006;3(2):376.

[49] Goldmeier D, Leiblum SR. Persistent genital arousal in women - a new syndrome entity. Int J STD AIDS. 2006;17(4):215-6.

[50] Mahoney S, Zarate C. Persistent sexual arousal syndrome: a case report and review of the literature. J Sex Marital Ther. 2007;33(1):65-71.

[51] Leiblum SR, Goldmeier D. Persistent genital arousal disorder in women: case reports of association with anti-depressant usage and withdrawal. J Sex Marital Ther. 2008;34(2):150-9.

[52] Waldinger MD, Venema PL, van Gils AP, Schweitzer DH. New insights into restless genital syndrome: static mechanical hyperesthesia and neuropathy of the nervus dorsalis clitoridis. J Sex Med. 2009;6(10):2778-87.

[53] Eibye S, Jensen HM. Persistent genital arousal disorder: confluent patient history of agitated depression, paroxetine cessation, and a tarlov cyst. Case Rep Psychiatry. 2014;2014:529052.

[54] de Magalhães FJ, Kumar MT. Persistent genital arousal disorder following selective serotonin reuptake inhibitor cessation. J Clin Psychopharmacol. 2015;35(3):352-4.

[55] Jackowich R, Pink L, Gordon A, Poirier É, Pukall CF. Symptom Characteristics and Medical History of an Online Sample of Women Who Experience Symptoms of Persistent Genital Arousal. J Sex Marital Ther. 2018;44(2):111-26.

[56] FDA. Questions and Answers: Finasteride Label Changes [Internet]. Available from: https:// wayback.archive-it.org/7993/20170723090425/https:/www.fda.gov/Drugs/DrugSafety/ InformationbyDrugClass/ucm299754.htm. Accessed 2018 February 19. 